\title{
Evaluation of Polymer Solar Cells Efficiency to Understand the Burn-In Loss
}

Arul Varman Kesavan, Khadija Kanwal Khanum, Saravanan Subbiahraj, Praveen C Ramamurthy*

Department of Materials Engineering, Indian Institute of Science, Bangalore-560012, India.

*Corresponding author and E-mail id: onegroupb203@gmail.com
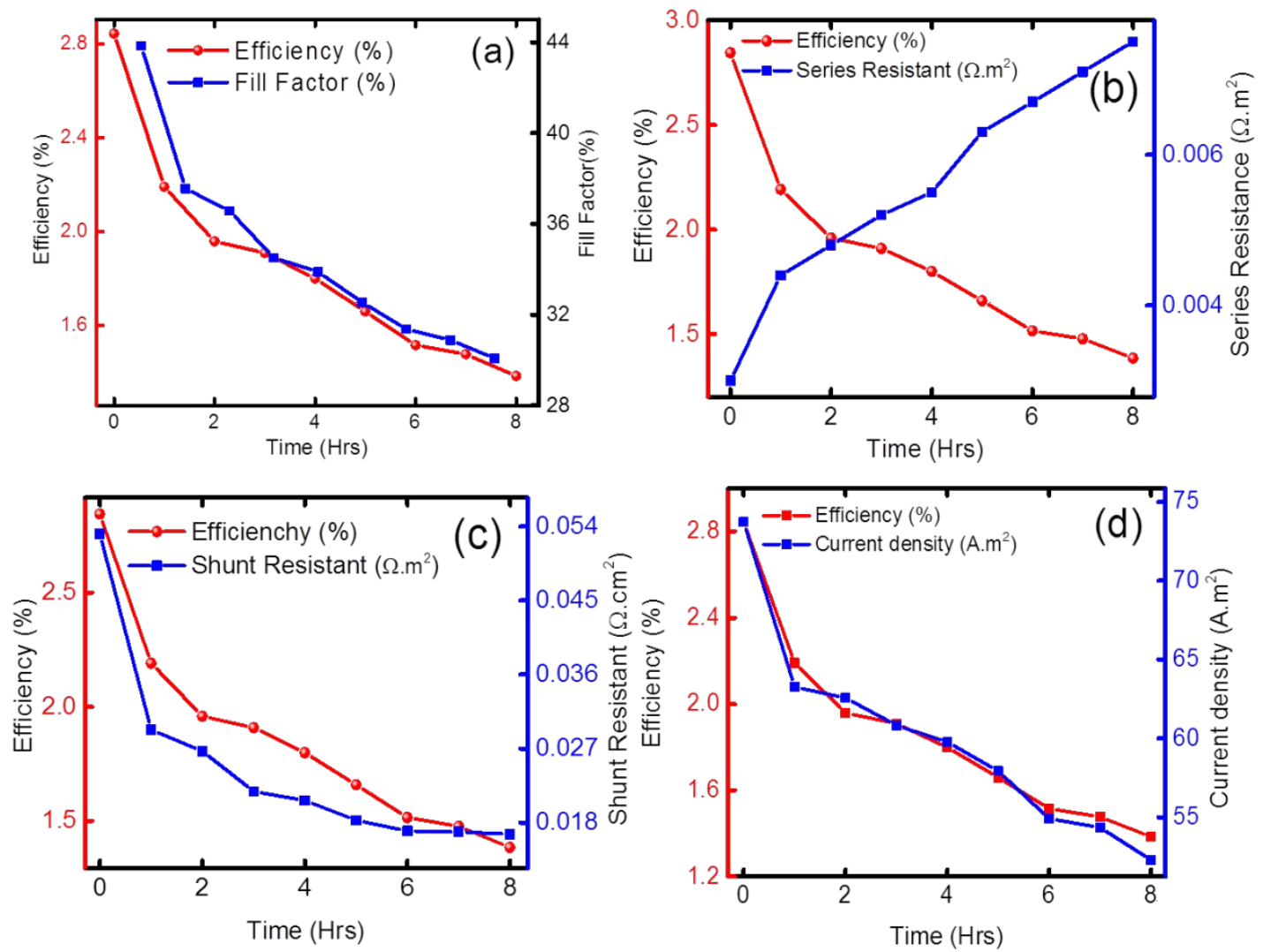

Figure S1. variation of efficiency with (a) fill factor, (b) series resistance, (c) shunt resistance, and (d) $\mathrm{J}_{\mathrm{SC}}$. 


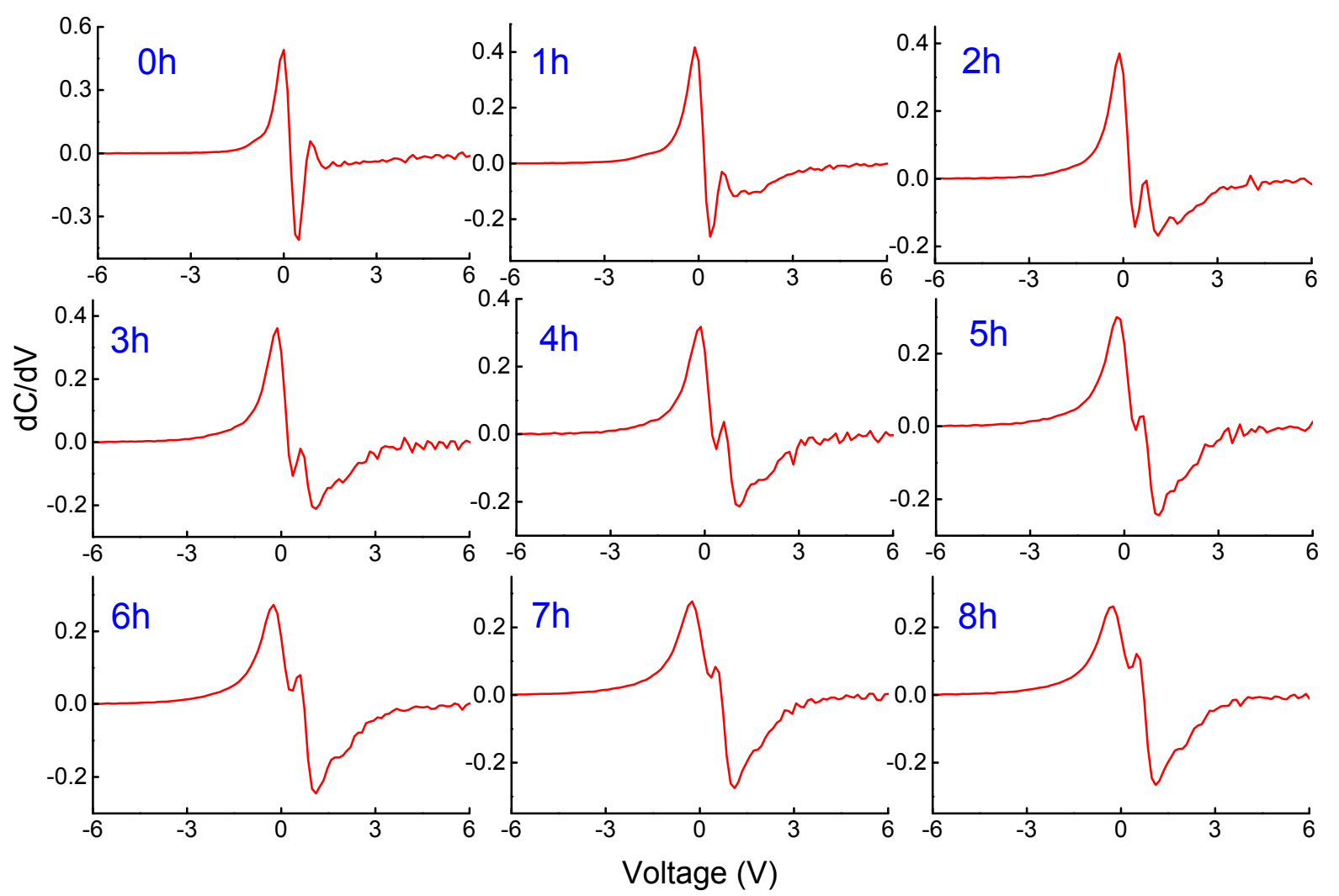

Figure S2. The derivative of capacitance-voltage characteristics of device under light (0-8h). 


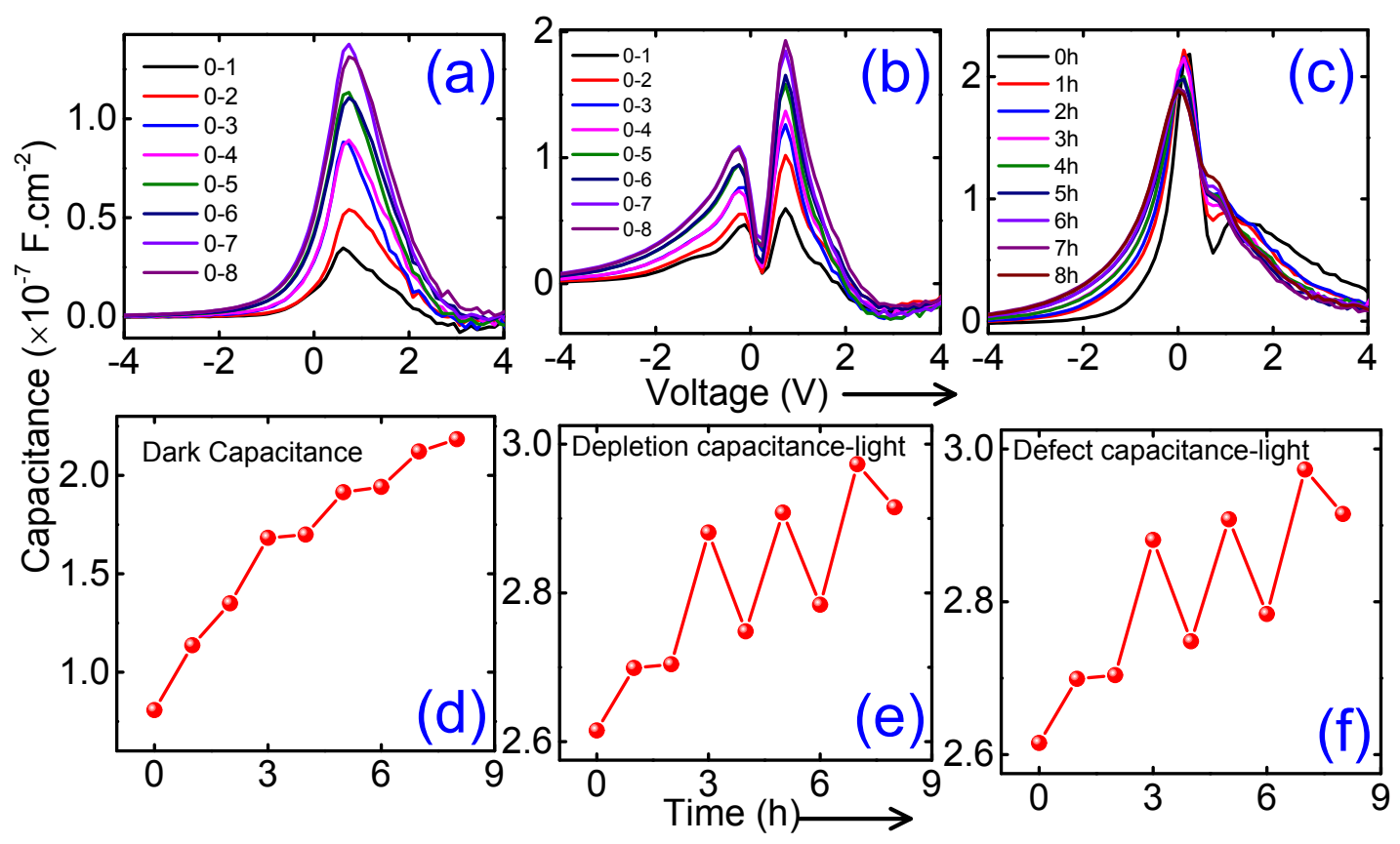

Figure S3. spectral representation of change in capacitance under (a) dark condition, (b) light condition, (c) difference in the capacitance between dark and light illuminated conditions for 0h$8 \mathrm{~h}$, with respect to ageing time (d) variation of peak value of dark capacitance, (e) variation of peak value of depletion capacitance under light, (f) variation of peak value of defect capacitance under light.

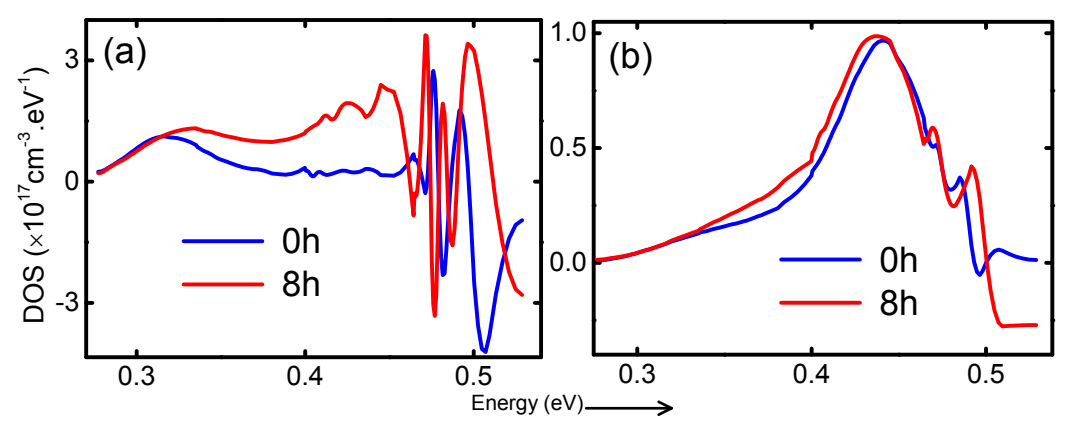

Figure S4. Device density of trap states distribution (0 and $8 \mathrm{~h})$ under (a) dark and (b) light conditions. 

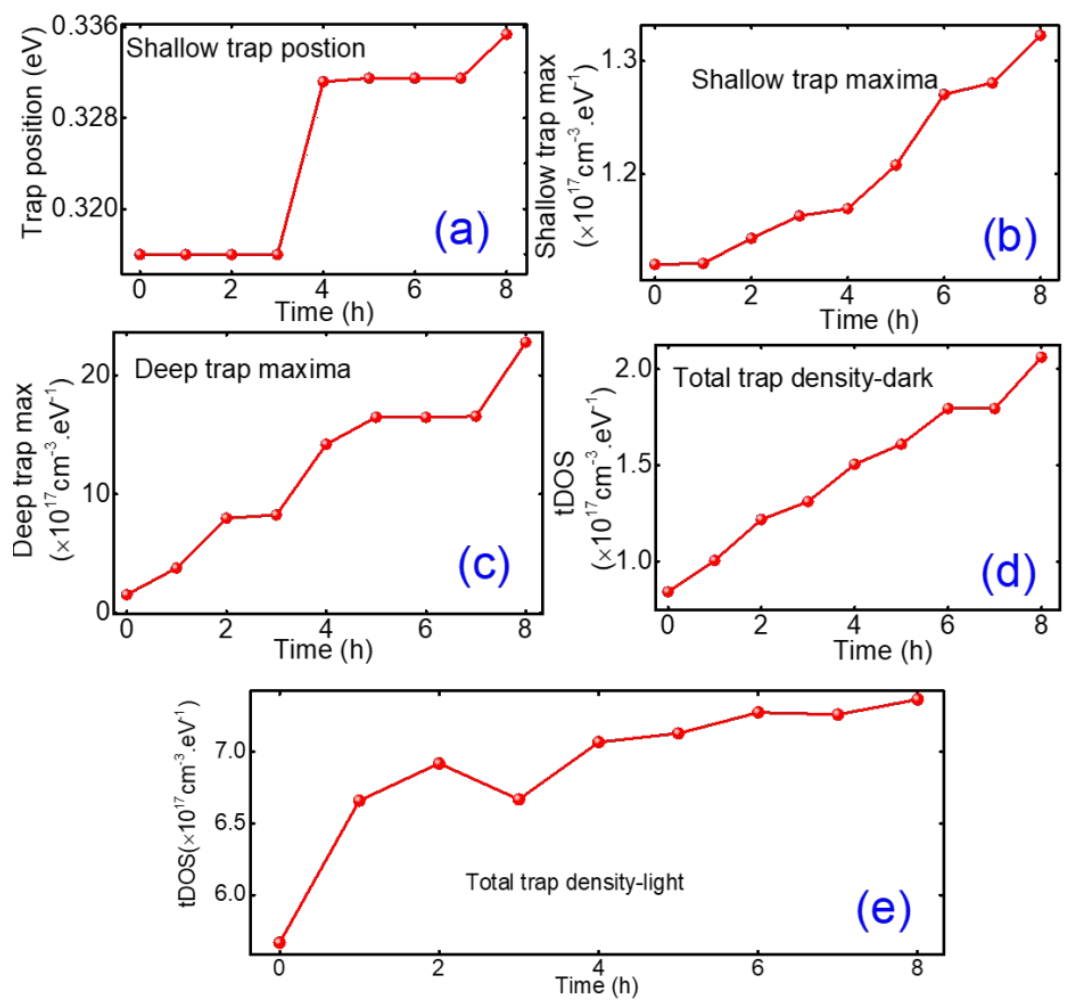

Figure S5. With respect to ageing time (a) variation in the energetic position of the shallow trap peak, (b) variation in the energetic position of the shallow trap peak maxima, (c) variation in the position of the deep trap maxima, (d) variation in the total density of trap states under dark and (e) variation in the total density of trap states under light. 

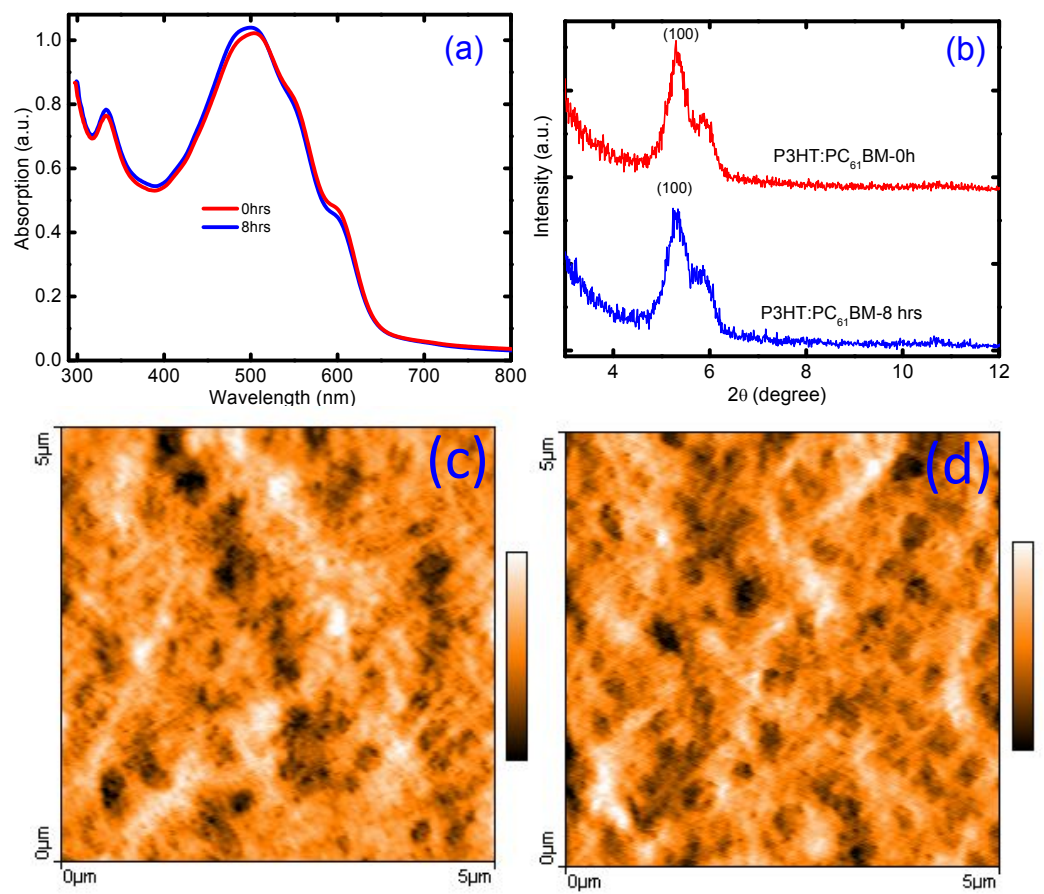

Figure S6. (a) UV-visible spectra, (b) GI-XRD spectra of P3HT:PC ${ }_{61} \mathrm{BM}$ active layer, AFM surface topography of $\mathrm{P} 3 \mathrm{HT}: \mathrm{PC}_{61} \mathrm{BM}(\mathrm{c})$ as prepared device $(0 \mathrm{~h})$ and $(\mathrm{d})$ after $8 \mathrm{~h}$ of continuous illumination. 
Table S1. Diode current density.

\begin{tabular}{cccccccccc}
\hline & $\mathbf{0}$ & $\mathbf{1}$ & $\mathbf{2}$ & $\mathbf{3}$ & $\mathbf{4}$ & $\mathbf{5}$ & $\mathbf{6}$ & $\mathbf{7}$ & $\mathbf{8}$ \\
\hline dark RB(1V) & 0.0851 & 0.2412 & 0.2248 & 0.2604 & 0.1702 & 0.3624 & 0.4661 & 0.4261 & 0.5553 \\
Dark FB(1V) & 4.6726 & 4.444 & 4.4173 & 4.4013 & 4.414 & 4.312 & 4.2907 & 4.2310 & 4.2293 \\
\hline
\end{tabular}

Table S2. Photovoltaic parameters.

\begin{tabular}{|c|c|c|c|c|c|c|}
\hline $\begin{array}{c}\text { Time } \\
(\mathrm{h})\end{array}$ & $\begin{array}{c}\mathrm{J}_{\mathrm{SC}} \\
\left(\mathrm{A} . \mathrm{m}^{-2}\right)\end{array}$ & $\begin{array}{c}\mathrm{V}_{\mathrm{OC}} \\
(\mathrm{mV})\end{array}$ & $\begin{array}{c}\mathrm{FF} \\
(\%)\end{array}$ & $\eta(\%)$ & $\begin{array}{c}\mathrm{R}_{\mathrm{s}} \\
\left(\Omega . \mathrm{m}^{2}\right)\end{array}$ & $\begin{array}{c}\mathrm{R}_{\text {sh }} \\
\left(\Omega . \mathrm{m}^{2}\right)\end{array}$ \\
\hline 0 & 73.730 & 554 & 43.05 & 1.746 & 0.0030 & 0.0531 \\
\hline 1 & 63.266 & 554 & 37.56 & 1.283 & 0.0044 & 0.0293 \\
\hline 2 & 62.828 & 554 & 36.06 & 1.196 & 0.0048 & 0.0267 \\
\hline 3 & 60.565 & 554 & 34.28 & 1.202 & 0.0052 & 0.0218 \\
\hline 4 & 59.770 & 554 & 33.45 & 1.104 & 0.0055 & 0.0207 \\
\hline 5 & 57.930 & 554 & 31.83 & 1.018 & 0.0063 & 0.0183 \\
\hline 6 & 54.902 & 554 & 31.11 & 0.931 & 0.0067 & 0.0170 \\
\hline 7 & 54.353 & 554 & 30.47 & 0.907 & 0.0071 & 0.0169 \\
\hline 8 & 52.293 & 554 & 30.09 & 0.849 & 0.0075 & 0.0166 \\
\hline
\end{tabular}


Table S3. Capacitance-voltage parameter.

\begin{tabular}{|c|c|c|c|c|}
\hline \multirow{2}{*}{$\begin{array}{l}\text { Time } \\
\text { (h) }\end{array}$} & \multicolumn{2}{|c|}{ Dark } & \multicolumn{2}{|l|}{ Light } \\
\hline & $\begin{array}{c}\mathrm{N} \\
\left(\mathrm{x} 10^{17} \mathrm{~cm}^{-3}\right)\end{array}$ & $\begin{array}{c}\mathrm{V}_{\mathrm{bi}} \\
(\mathrm{mV})\end{array}$ & $\left(\begin{array}{c}\mathrm{N} \\
\left(\mathrm{x} 10^{16} \mathrm{~cm}^{-3}\right)\end{array}\right.$ & $\begin{array}{c}\mathrm{V}_{\mathrm{bi}} \\
(\mathrm{mV})\end{array}$ \\
\hline 0 & 1.984 & 554 & 2.547 & 500 \\
\hline 1 & 3.142 & 523 & 2.843 & 470 \\
\hline 2 & 3.129 & 523 & 2.917 & 460 \\
\hline 3 & 3.565 & 521 & 3.417 & 450 \\
\hline 4 & 3.795 & 520 & 3.641 & 440 \\
\hline 5 & 4.384 & 515 & 3.785 & 440 \\
\hline 6 & 4.479 & 514 & 3.914 & 440 \\
\hline 7 & 4.765 & 512 & 3.996 & 430 \\
\hline 8 & 5.173 & 511 & 4.125 & 430 \\
\hline
\end{tabular}

Table S4. Capacitance-voltage parameters.

\begin{tabular}{|c|c|c|c|c|c|c|c|}
\hline \multirow{2}{*}{$\#$} & \multicolumn{2}{|c|}{ Device tested under dark } & \multicolumn{5}{|c|}{ Device tested under light } \\
\cline { 5 - 9 } & \multirow{2}{*}{ FWHM } & $\begin{array}{c}\mathrm{V}_{\text {peak }} \\
(\mathrm{V})\end{array}$ & $\begin{array}{c}\mathrm{C} \\
\left(\times 10^{-7} \mathrm{Fm}^{-2}\right)\end{array}$ & $\begin{array}{c}\mathrm{V}_{\text {peak }} \\
(\mathrm{V})\end{array}$ & $\begin{array}{c}\mathrm{C} \\
\left(\times 10^{-7} \mathrm{Fm}^{-2}\right)\end{array}$ & $\begin{array}{c}\mathrm{V}_{\text {peak }} \\
(\mathrm{V})\end{array}$ & $\begin{array}{c}\mathrm{C} \\
\left(\times 10^{-7} \mathrm{Fm}^{-2}\right)\end{array}$ \\
\hline 0 & 1.0605 & 0.7067 & 0.8073 & 0.275 & 2.615 & 1.50 & 1.478 \\
\hline 1 & 1.2663 & 0.7364 & 1.1365 & 0.1615 & 2.699 & 0.85 & 1.945 \\
\hline 2 & 1.3216 & 0.7364 & 1.3493 & 0.1538 & 2.704 & 0.81 & 2.383 \\
\hline 3 & 1.2352 & 0.7364 & 1.6818 & 0.1981 & 2.881 & 0.80 & 2.627 \\
\hline 4 & 1.3532 & 0.7364 & 1.6993 & 0.1881 & 2.748 & 0.74 & 2.734 \\
\hline 5 & 1.3405 & 0.7364 & 1.9142 & 0.1800 & 2.908 & 0.68 & 2.946 \\
\hline 6 & 1.4230 & 0.7364 & 1.9411 & 0.1756 & 2.784 & 0.74 & 3.019 \\
\hline 7 & 1.3849 & 0.7364 & 2.1208 & 0.2278 & 2.973 & 0.72 & 3.209 \\
\hline 8 & 1.4838 & 0.7666 & 2.1833 & 0.1627 & 2.915 & 0.74 & 3.2937 \\
\hline
\end{tabular}


Table S5. Parameter of density of trap states.

\begin{tabular}{|c|c|c|c|c|c|}
\hline $\begin{array}{c}\text { Time } \\
(\mathrm{h})\end{array}$ & $\begin{array}{c}\text { Shallow trap } \\
\text { peak position } \\
(\mathrm{eV})\end{array}$ & $\begin{array}{c}\text { Shallow trap peak } \\
\text { intensity }\left(\mathrm{T}_{\mathrm{W}}\right) \\
\left(10^{17} \mathrm{~cm}^{-3} \mathrm{eV}^{-1}\right)\end{array}$ & $\begin{array}{c}\text { Deep trap peak } \\
\text { intensity } \\
(\text { at Ew-0.4487eV }) \\
\left(10^{16} \mathrm{~cm}^{-3} \mathrm{eV}^{-1}\right)\end{array}$ & $\begin{array}{c}\text { Total trap DOS } \\
\left(\mathrm{cm}^{-3} \mathrm{eV}^{-1}\right) \\
\left(10^{16} \mathrm{~cm}^{-3} \mathrm{eV}^{-1}\right) \\
(\mathrm{dark})\end{array}$ & $\begin{array}{c}\text { Total trap DOS } \\
\left(\mathrm{cm}^{-3} \mathrm{eV}^{-1}\right)\left(10^{15} \mathrm{c}\right. \\
\left.\mathrm{m}^{-3} \mathrm{eV}^{-1}\right) \\
(\text { light })\end{array}$ \\
\hline 0 & 0.31597 & 1.1202 & 1.53894 & 0.8421 & 5.6671 \\
\hline 1 & 0.31597 & 1.1211 & 3.77547 & 1.0054 & 6.6572 \\
\hline 2 & 0.31597 & 1.1433 & 7.97135 & 1.2180 & 6.9161 \\
\hline 3 & 0.31597 & 1.1631 & 8.25632 & 1.3115 & 6.6656 \\
\hline 4 & 0.33120 & 1.1695 & 14.1996 & 1.5051 & 7.0642 \\
\hline 5 & 0.33150 & 1.2079 & 16.4659 & 1.6091 & 7.1256 \\
\hline 6 & 0.33150 & 1.2704 & 16.4659 & 1.7947 & 7.2712 \\
\hline 7 & 0.33150 & 1.2804 & 16.5532 & 1.7948 & 7.2565 \\
\hline 8 & 0.33538 & 1.3225 & 22.7831 & 2.0614 & 7.3624 \\
\hline
\end{tabular}

